\title{
Norois
}

Environnement, aménagement, société

$217 \mid 2010 / 4$

Interroger les processus de valorisation des espaces urbains

\section{Contribution aux réflexions sur la spatialisation de la valeur : retour sur un colloque}

Categorizations of space and appropriations of places: a contribution to the studies of the spatial manifestations of the value

\section{Régis Keerle}

\section{(2) OpenEdition \\ Journals}

\section{Édition électronique}

URL : http://journals.openedition.org/norois/3490

DOI : $10.4000 /$ norois.3490

ISBN : 978-2-7535-1565-9

ISSN : $1760-8546$

Éditeur

Presses universitaires de Rennes

Édition imprimée

Date de publication : 15 décembre 2010

Pagination : 91-108

ISBN : 978-2-7535-1340-2

ISSN : 0029-182X

Référence électronique

Régis Keerle, "Contribution aux réflexions sur la spatialisation de la valeur : retour sur un colloque », Norois [En ligne], 217 | 2010/4, mis en ligne le 31 décembre 2012, consulté le 19 avril 2019. URL http://journals.openedition.org/norois/3490; DOI : 10.4000/norois.3490 


\title{
CONTRIbUtion AUX RÉfLEXIONS \\ SUR LA SPATIALISATION DE LA VALEUR : \\ RETOUR SUR UN COLLOQUE
}

\author{
RÉGis KeERLE \\ ESO-Rennes - UMR 6590 ESO CNRS \\ (Université Rennes 2), \\ Laboratoire ESO-Rennes, Maison de la recherche en sciences sociales, \\ Square Henri-Le-Moal - 35043 Rennes Cedex, France \\ regis.keerle@univ-rennes2.fr
}

\begin{abstract}
RÉSUMÉ
Ce texte se base sur les communications présentées lors du colloque "Espaces hérités, espaces enjeux : appropriations, (dé)valorisations, catégorisations "(Caen, 2007). Leur lecture nourrit une réflexion sur la spatialisation de la valeur qui révèle des différences de traitements théoriques et disciplinaires de cette question. Notre réflexion, inscrite dans le cadre de la géographie sociale, s'appuie sur la notion de réification. Elle nous permet de proposer un mode de qualification temporalisé des actions témoignant de la dimension spatiale des sociétés.
\end{abstract}

\section{MOTS CLÉ : action - espace - réification - spatialisation - valeur - temporalités}

\section{ABSTRACT}

Categorizations of space and appropriations of places: a contribution to the studies of the spatial manifestations of the value

This text is based on the papers presented at the conference "Inherited spaces, contested spaces: appropriations, increase standing or depreciation of space, categorizations" (Caen, 2007). These papers give rise to a reflexion on the process underlying the spatial manifestations of the value. We pinpoint theoretical and disciplinary differences in the examination of this question. Within the framework of social geography, our reflexion relies on the concept of reification. It allows us to suggest a model including a time perspective in qualification of the actions revealing the spatial dimensions of societies.

KEY WORDS : action - reification - space - spatial manifestations - value - time

Le colloque de Caen intitulé «Espaces hérités, espaces enjeux : appropriations, (dé)valorisations, catégorisations » (2007) a mis en valeur des travaux de recherche dont une trentaine, sélectionnés par son comité scientifique, sont publiés ou en cours de publication dans différentes revues (dont ce numéro de Norois) et un ouvrage. Membre de ce comité, nous avons pu vérifier l'unité thématique de ce colloque grâce à la forme de son organisation (absence d'ateliers spécifiques et mise à disposition des versions écrites des communications présentées), au-delà de la diversité des supports de publication de ces travaux. Nous proposons, pour rendre compte de 
cette unité, une lecture transversale, axée sur la question de la réification des valeurs spatiales, des textes ainsi sélectionnés ${ }^{1}$.

Afin d'apprécier dans cette perspective les apports de ce colloque pluridisciplinaire, nous les mettrons en regard avec des éléments du paysage scientifique de la géographie qui leur correspondent, tels que décrits par le Dictionnaire de la géographie et de l'espace des sociétés. Celui-ci rappelle qu'il faut se garder de "verser dans la naturalisation des valeurs spatiales et dans la croyance en leur caractère anhistorique » (Lussault, 2003a) et définit la valeur spatiale comme « l'expression des substances sociétales qu'un espace recèle... La question de la valeur renvoie donc à l'analyse des conditions sociétales générales et à la condensation dans des agencements spatiaux de valeurs (positives et/ou négatives) par les acteurs sociaux ». M. Lussault approfondit cette définition à l'aide d'un exemple pris dans le marché immobilier, exemple où la mention d'une construction et d'une «stabilisation de la valeur spatiale » (Lussault, 2003a) ne débouche pas sur une réflexion relative à la temporalité des constructions spatiales et des valeurs qui leur sont attribuées. Nous en concluons rapidement qu'il existe un « déficit d'intelligibilité » (Berthelot, 1996) dans la conception de la valeur, appliquée en géographie, présentée par cet auteur, et qu'il est légitime de contribuer à combler ce vide par une réflexion basée sur le principe épistémologique du pluralisme explicatif. Ce dernier peut être décrit ici comme la mise en discussion, par des programmes de recherche (Berthelot, 2001)2 ${ }^{2}$, des approches statiques ou dynamiques de plusieurs types d'êtres (ici, acteurs ou agents, formes spatiales), d'états (ici, positions sociale et spatiale des acteurs ou agents) et de processus (ici, appropriation, catégorisation, valorisation) appréhendés à divers niveaux d'analyse (échelles de généralité, de temps et de masse) de la spatialisation des rapports sociaux (Grossetti, 2006).

Cette réflexion s'appuie sur les versions des textes fournies par leurs auteurs avant leur publication. Notre positionnement épistémologique, dont nous montrerons l'intérêt par l'exemple plutôt que par une logique déductive, nous conduit à revendiquer un ancrage en géographie sociale. Il servira surtout notre interprétation de ces textes, dont nous nous efforcerons de respecter la spécificité. Notre principal développement théorique nous amènera à proposer une nouvelle interprétation de l'articulation entre action, formes spatiales et temporalités en géographie.

L'organisation de notre propos peut être appréhendée à l'aide du schéma en annexe (fig. 1). Sa première séquence est consacrée à l'explicitation de son positionnement épistémologique. Le rapport de notre analyse aux formes spatiales y sera seulement suggéré, l'accent étant mis d'abord sur un exemple de la diversité disciplinaire de la prise en compte de la dimension spatiale de l'action en sciences anthroposociales, puis sur une première caractérisation du langage d'interprétation de géographie sociale que nous employons pour présenter notre lecture des textes du colloque. Sa deuxième séquence constitue une mise en application de ce positionnement épistémologique, sélectionnant dans ces textes les éléments d'une lecture des relations sociales suffisamment générique (espace social, acteurs et agents, discours et pratiques) pour accueillir cette diversité sans chercher à la réduire. Elle débouche sur une proposition de catégorisation des effets des actions (stabilisation ou subversion) sur les formes spatiales. Cette catégorisation conduit dans la dernière séquence à une interrogation sur la notion de réification, dont nous proposons une explicitation nouvelle en géographie, articulée avec une analyse des liens entre temporalités et spatialités.

1. La liste des textes placée en annexe indique, pour ceux d'entre eux encore en cours d'évaluation, les supports de publication sollicités. Certaines communications sélectionnées par le comité scientifique du colloque, essentielles pour notre problématique, n'ont pu être présentées par leurs auteurs à des fins de publication : leur titre a été mentionné en complément de la référence de ces auteurs qui leur était conceptuellement la plus proche lorsque cela était possible.

2. Les programmes de recherche peuvent être résumés par les choix opérés au sein d'une combinaison de schèmes d'explication. Le schème actanciel suppose ainsi de recourir à un moment donné de l'explication aux intentions d'un individu pour expliquer un phénomène qui le concerne. Ce recours aux schèmes explicatifs, qui permet de ne pas réduire, par exemple, les raisonnements sociologiques à une opposition entre « holisme » et « individualisme » ou toute autre forme de dualisme, ouvre la voie à un pluralisme explicatif. Les travaux de J.-M. Berthelot (1945-2006) ont été discutés lors d’une journée d'étude organisée en son hommage [http://w3.lisst.univ-tlse 2.fr/journee_etude/jberthelot.htm]. 


\section{Diversité des expressions, confrontation sereine des programmes d'analyse}

L'intérêt porté par de nombreuses disciplines à la thématique du colloque, suscité par la diversité disciplinaire des membres de son comité scientifique ${ }^{3}$, interroge la géographie : quels apports peut-elle en attendre? La mise en regard du texte d'une sociologue avec une des orientations de la géographie sociale fournira un exemple de cet enrichissement potentiel.

A propos des rapports entre disciplines, J.-M. Berthelot relève la persistance de leurs noyaux fondateurs (à notre sens, les formes spatiales, pour la géographie française), donc de leurs différences, ainsi que leurs dynamiques d'expansion, donc potentiellement de rencontre (comme lors du colloque), et surtout le caractère transversal de leurs structurations internes par des constructions théoriques de plus ou moins large portée, mais assez rarement limitées à une seule discipline (Berthelot, 2001). Ce type de réflexion sur les relations entre disciplines n'est pas encore très développé en géographie. L'emploi de l'expression « substances sociétales » contenue dans la définition précitée de la notion de valeur spatiale renvoie ainsi implicitement à la notion de substance, définie par J. Lévy comme « composante non spatiale d’une configuration spatiale » (Lévy, 2003a) et introduite dans le vocabulaire de la géographie pour l'inciter à "s'intéresser à des aspects qui semblent sortir de son champ ", jusqu'à pratiquer, le cas échéant, une " géographie thématique » (Lévy, 2003a). Mais cette notion de substance reste imprécise sur le plan épistémologique, le terme «thématique » mêlant ici des relations interdisciplinaires (géographie et économie...) et des domaines intradisciplinaires (géographie de l'industrie...) sans mentionner l'existence d'alternatives théoriques qui les traversent. Or cette conception de la structuration interne de la géographie et de sa position parmi les sciences anthroposociales a des conséquences sur l'appréciation par J. Lévy de l'existence du courant de la géographie sociale. Notre justification épistémologique de la diversité des orientations théoriques des textes du colloque permet d'argumenter en faveur de la pertinence de cette existence.

\section{UN COLLOQUE PLURIDISCIPLINAIRE,}

\section{DES LECTURES DIFFÉRENTES DE LA DIMENSION SPATIALE DES SOCIÉTÉS}

Géographie, anthropologie, histoire, science politique, sociologie, constituent des filtres disciplinaires (fig. 1) ${ }^{4}$, auxquels s'ajoutent des spécialisations transversales (sciences de l'éducation, urbanisme), qui contribuent à expliquer les choix de supports de publication effectués pour les textes du colloque retenus à cet effet. Ces filtres disciplinaires sont autant de cadres de structuration des données qui peuvent devenir, si l'on n'y prend garde, sources de malentendus entre disciplines ou prétextes à des enfermements thématiques aussi confortables qu'efficaces. C'est le cas du «capital d'autochtonie » (Mazaud, 2010), concept d'origine sociologique qui fait écho pour le géographe à « l'acteur endogène » (Di Méo, 1987). Rappelons d'abord la signification de chacune de ces expressions avant d'interroger l'absence de leur confrontation puis de proposer un exemple de dialogue entre ces deux disciplines.

La notion d'acteur endogène est employée par G. Di Méo pour qualifier l'une des « deux catégories génériques d'acteurs [qui] s'approprient l'espace selon des modalités et en fonction d'enjeux qui reflètent conjointement leur personnalité propre et leur position dans les rapports de classes ou de groupes » (Di Méo, 1987). Elle caractérise l'individu « qui s'implique de l'intérieur dans une formation socio-spatiale » (Di Méo, 1987), à l'inverse de la catégorie d'acteur exogène, qualifiant la situation dans laquelle «l'acteur social se contente d'envisager l'espace comme un produit, un champ d'action et un enjeu » (Di Méo, 1987). Du côté de la sociologie, pour bénéficier d'un capital d'autochtonie, «l'essentiel est d'appartenir au réseau de parenté ou de sociabilité d'un

3. Les contributions des géographes ne représentent qu’à peine plus de la moitié des textes retenus pour publication.

4. Les expressions ou termes en italique renvoient à la fig. 1 . 
membre du groupe travaillant au sein de l'organisation économique dominante de la commune et d'y être visible localement » (Mazaud, 2010) La proximité entre les conceptions de G. Di Méo et celles de C. Mazaud, exprimées dans son étude de la transmission d'entreprises artisanales en zone rurale, est très nette dans l'extrait qui suit : "l'usage du capital d'autochtonie freine ainsi l'arrivée de repreneurs extérieurs au territoire, même fortement dotés en capitaux économique et culturel, venus acheter une entreprise en zone rurale pour y "exploiter" les savoir-faire et les atouts locaux, en ne participant ni à l'effort ni à la vie de la commune » (Mazaud, 2010). Pourtant, cette analyse sociologique en termes de résistance de l'artisanat de métier (ici, en électricité, plomberie et chauffage) face à l'arrivée de gestionnaires d'entreprises employant des salariés qualifiés ignore les propositions du géographe précité.

C. Mazaud (2010) rappelle l'origine du concept de capital d'autochtonie (daté de 1980), mais signale aussi la rareté de son emploi ultérieur en sociologie avant les années 2000, rareté dont les causes restent à expliquer. Parmi celles-ci, nous avançons l'hypothèse de la faiblesse des dialogues interdisciplinaires, et ici particulièrement entre sociologie et géographie. En effet, G. Di Méo fait explicitement référence à sa lecture de travaux de M. Bozon (1984), auteur signalé par C. Mazaud comme co-promoteur du concept de capital d'autochtonie (Bozon et Chamboredon, 1980). Par contre, lorsque ce concept est employé par la suite en sociologie, c'est sans aucune mention de l'emploi de celui d'acteur endogène en géographie. Faut-il y voir une dette absolue de la géographie face à la sociologie ou y a-t-il ici un exemple d'espace de connaissance potentiellement interdisciplinaire à explorer? Une remarque de C. Mazaud (2010) relative à la notion de territoire («c'est en observant attentivement les phénomènes sociaux qui se déroulent en son sein qu'il [le territoire] ne se donne plus à voir comme le simple cadre géographique entourant un événement »), témoignant de sa relative méconnaissance de notre discipline, nous laisse penser que la seconde hypothèse est la plus féconde. La rapide comparaison qui suit vise à l'expliciter.

La notion de capital d'autochtonie est plus précise que celle d'acteur endogène, car ce type d'acteurs peut, ou non, être détenteur de ce capital selon la définition que l'on donne de son contenu. Ainsi, seuls quelques habitants d'un type de localité tel que le petit bourg de l'ouest de la France étudié par C. Mazaud (2010) peuvent être détenteurs d'un capital d'autochtonie permettant de transmettre une entreprise artisanale. Et la généralisation, en géographie, de cette qualification des différents types de capitaux d'autochtonie selon les types de localité resterait à entreprendre. Par contre, l'identification des situations d'obsolescence ou de résilience de ces capitaux peut trouver dans la notion de formation socio-spatiale, entendue comme outil méthodologique, un étayage utile à la formulation d'hypothèses de travail relatives à cette question. En invitant à considérer les différents domaines de l'activité sociale et les différentes échelles auxquelles sont confrontés les individus impliqués dans ces domaines et au sein desquels ils peuvent constituer leurs ressources, cette notion permet de concevoir l'extension spatiale de ces ressources porteuses de différenciations géographiques. La dimension spatiale de la valeur contenue dans la connaissance de la clientèle par l'artisan et la confiance qu'elle accorde à cet acteur endogène qui se dévoue à elle, valeur que cet artisan, à l'heure de la retraite, refuse de transmettre à un acteur exogène, n'est alors qu'un exemple de la diversité de l'extension spatiale des ressources individualisées. La multiplication des analyses des situations de valorisation de ces ressources pourrait aboutir à une complexification des qualifications des positions des acteurs et des échelles spatiales de leurs ressources permettant de renforcer la pertinence de leurs interprétations théoriques et de préciser leurs limites. Car la posture du pluralisme explicatif suppose le renoncement à la réduction de l'incertitude de la connaissance par la proclamation d'une théorie intégratrice ${ }^{5}$ (Berthelot, 2001) autant qu'au cloisonnement des disciplines ou à la prétention de leur dépassement.

5. Par souci de concision, nous ne rappelons pas ici la réflexion sur la notion de valeur développée par la géographie structurale. Signalons simplement que celle-ci prend la forme d'une théorie intégratrice qui, comme d'autres avant elle, a révélé sa «polysémie programmatique »(Berthelot, 2001). 


\section{LA DIVERSITÉ DES ORIENTATIONS THÉORIQUES, CONDITION D'EXISTENCE DE LA GÉOGRAPHIE SOCIALE}

J. Lévy a récemment interrogé le positionnement incertain d'une géographie sociale qui serait, soit un pléonasme pour une géographie conçue comme science sociale de l'espace des sociétés, soit une géographie sociologique qui suppose " une prise de contact sérieuse et ouverte avec la sociologie [...] dotée d'un excellent outillage théorique » (Lévy, 2008). En réponse à cette invitation à l'approfondissement de la réflexion sur l'emploi de l'expression " géographie sociale », nous précisons ici notre interprétation de la diversité des orientations théoriques des textes du colloque.

Si nous considérons les théories comme des applications d'un programme de recherche « à un domaine de réalité défini dans une discipline déterminée » (Berthelot, 2001), il est logique de constater que le statut des références théoriques évoquées dans ces textes varie d'une part selon les disciplines de présentation de leurs auteurs et d'autre part selon leurs domaines intradisciplinaires d'étude, ces deux dimensions ne pouvant être entièrement séparées.

Pour le premier point, on remarquera ainsi classiquement la discrétion relative des références théoriques dans les textes présentés par les historiens ${ }^{6}$, typique d'une discipline dont la structure argumentative se distingue par le « primat de l'étayage empirique sur la logique déductive » (Berthelot, 1996). Cependant, cette discrétion du recours à la théorie dans l'explication ne signifie pas absence ou refus de positionnement. Ainsi, inscrire explicitement sa recherche dans le domaine de l'histoire sociale par l'étude de «la territorialisation des administrateurs coloniaux » (NavarroAndraud, à paraître) à Saint-Domingue au XVIII siècle pourrait s'apparenter, pour un géographe, à pratiquer une géographie «thématique » où la « substance » (le domaine) concernée serait le « social ». Toutefois, de même qu'en histoire, ce thème n'est pas considéré à valeur égale selon les différentes positions épistémologiques des historiens, en géographie, il ne suffit pas de dissoudre l'appellation « géographie sociale » dans l'appellation « géographie, science sociale de l'espace des sociétés » pour résoudre la question de l'appréciation de la validité des choix épistémologiques effectués par les géographes en amont de leur inscription disciplinaire pour traiter du « social ». Précisons donc que, sur ce point, le questionnement central du colloque interrogeait les rapports entre valeur des espaces et positions sociales. Dans la mesure où il s'agissait plus précisément de questionner le rôle tenu par l'appropriation, la catégorisation et la hiérarchisation des espaces dans la production des inégalités, la différenciation et la hiérarchisation des individus et des groupes sociaux, ainsi que dans la construction de rapports sociaux dissymétriques, des références à « l'école de sociologie incarnée par Pierre Bourdieu » (Lévy, 2003b) figurent dans ces contributions, que leurs auteurs soient géographes (Goeury et al., à paraître) ou non (Mazaud, 2010 ; Meslin, à paraître). Elles ne sont, de loin, ni majoritaires ni exclusives : certains auteurs se réfèrent ainsi à M. Foucault (Morelle, à paraître), d'autres à L. Boltanski et E. Chiapello (Vermeersch, à paraître)... Contrairement à ce qui est implicitement reproché à la géographie sociale, et dans la perspective d'un pluralisme explicatif, nous ne voyons dans ces références nulle volonté de nier le fait qu'il peut être « efficace de confronter, dans l'analyse, la notion de domination à ses contraires : coopération, égalité, justice ». Nous constatons simplement que ces contributions témoignent de la proximité de "géographes sociaux ${ }^{7}$ » avec d'autres disciplines, dont la sociologie, au moins pour certaines thématiques.

Pour le second point, remarquons que les orientations thématiques par domaines intradisciplinaires sont parfois privilégiées dans les textes ici analysés, qu'il s'agisse des processus de patrimonialisation (Gillot, à paraître) ou de gentrification (Guérois et Hancock, à paraître; Ter Minassian, ce numéro). Or, les liens entre choix d'objets d'étude et choix théoriques légitimes correspondants

6. Voir par exemple, dans un voisinage avec des géographes, pour l'évolution de la localisation des « espaces résidentiels élitaires à Bruxelles (XVIII ${ }^{e}-\mathrm{XXI}^{e}$ siècles) », Debroux, Decroly, Deligne, Galand, Loir et Van Criekingen (à paraître).

7. Des auteurs extérieurs à l'UMR ESO, co-organisatrice du colloque, affirment se référer à une géographie sociale (Sabatier, 2006) ou évoquent des auteurs s'y référant (Gillot et Morelle, à paraître). 
y transparaissent régulièrement : par exemple, les phénomènes migratoires ont été étudiés par l'école de Chicago, ce qui justifie son évocation par les travaux qui s'y consacrent (Dequirez, 2010). Il n'est donc pas possible de disqualifier a priori un courant d'analyse, interne à une discipline ou transdisciplinaire, sans disqualifier aussi, le plus souvent dans le même mouvement, ses objets d'étude, ce qui constitue une position épistémologique inconciliable avec la posture d'un pluralisme explicatif reconnaissant la validité de plusieurs programmes de recherche et la fécondité de leur confrontation réglée (Berthelot, 2001).

Par contre, il est toujours possible de reconnaître, grâce à cette posture pluraliste, la pertinence d'une proposition explicative nouvelle d'un phénomène déjà étudié. Ainsi, face à « une histoire générale des villes du maghreb qui a le plus souvent considéré la domination de l'administration coloniale comme une évidence dont il convenait d'étudier uniquement les modes d'expression », a-t-il été montré, à partir du cas de Tripoli (Libye) pendant la colonisation italienne, qu'une analyse basée sur un « modèle de ville binaire (opposant la ville européenne à la médina ou ville indigène) [... érigé en paradigme d'un urbanisme uniquement au service du pouvoir colonial [...] ne permet pas de saisir la complexité des facteurs sociaux et des processus de décision dans l'instauration d'une géographie urbaine liée à la colonisation » (Dumasy, à paraître). À l'image de cette analyse qui réfute une interprétation simpliste des phénomènes de domination en mettant en valeur l'importance du contexte spatial dans lequel ils s'exercent, nous nous efforcerons de limiter la portée des montées en généralité que nous proposerons depuis les textes du colloque à un langage d'interprétation qui permet leur mise en dialoque avec notre positionnement en géographie sociale.

\section{Proposition d'une lecture unitaire du colloque}

La poursuite de notre lecture des apports du colloque à la réflexion sur la spatialisation de la valeur regroupe l'analyse des diverses manifestations de sa construction au sein de deux thématiques et de deux formes de qualification des données construites et interprétées dans ceux de ses textes ici retenus.

La première thématique, celle de l'espace social, est plus typique d'une approche de géographie sociale que la seconde, laquelle interroge les parcours des individus ou par extension, des groupes sociaux, dans cet espace à l'aune d'une de leurs qualifications possibles issues de la sociologie. Leur distinction ne vise qu’à illustrer la possibilité qu'offrent les contributions de mettre l'accent, soit sur la segmentation et la hiérarchisation de l'espace social dont témoigne l'existence de formes spatiales appropriées et inégalement valorisées, soit sur l'absence de déterminisme absolu pesant sur les individus amenés à fréquenter ces formes.

Les deux formes de qualification des données choisies sont moins marquées par des orientations théoriques ou disciplinaires. Leur distinction entre discours et pratiques relatifs aux formes spatiales vise, d'une part, à mettre en évidence les différences de capacités d'action des individus dans l'espace et sur l'espace, d'autre part à l'introduction de notre conception de la temporalité des constructions spatiales et des valeurs qui leur sont attribuées.

\section{DES AGENTS ET DES ACTEURS ARPENTANT L'ESPACE SOCIAL}

L'emploi de l'expression « espace social » est ici à prendre assez simplement dans le sens de l'espace des positions sociales et des formes spatiales qui en résultent. Quant à sa signification sociologique, cet emploi ne vise pas à réduire l'analyse du social à celle des positions sociales, mais seulement à considérer qu'il s'agit d'une de ses voies d'analyse légitimes, parmi d'autres, et qu'elle a été illustrée, selon notre lecture, dans les textes évoqués ici. La référence aux deux sens du mot «arpenter », qui combinent le parcours d'une étendue et sa mesure, laquelle peut être le signe ou la trace d'une action passée ou à venir, suggère le rôle de ces actions dans la reproduction/ transformation de l'espace social dans le temps. 


\section{Espace social}

L'espace social, nécessairement mondial pour le géographe, est segmenté et les formes de spatialisation de ces segmentations se signalent à plusieurs échelles. Celles-ci peuvent être envisagées globalement (Monde, États, sociétés locales, groupements sociaux, individus...) ou traitées conjointement par définition d'un type de généralité («l'économie », «l'industrie »...) permettant de caractériser les formes spatiales d'une manière plus analytique. C'est à notre sens la démarche qu’a employée G. Di Méo (1987) pour forger le modèle de la formation socio-spatiale, dont nous avons relevé l'intérêt. Par contre, notre scepticisme quant à la pertinence de la distinction d'une instance géographique dans ce modèle nous amène à éviter de reprendre les catégories analytiques qui l'ont inspiré pour expliciter ici rapidement notre conception de l'espace social. Nous nous limiterons donc à évoquer dans leur perspective les occurrences politiques les plus directes de ces segmentations illustrées par les textes du colloque.

Emblématiques dans le cas du «mur de séparation entre israëliens et palestiniens » (Guillot, à paraître), les effets de la segmentation étatique de l'espace mondial sont mis en évidence dans plusieurs contributions, comme dans l'analyse des formes de «l'appropriation de la mer dans le golfe Normand-Breton », comprenant les îles anglo-normandes (Fleury, à paraître). L'illustration de la prégnance des cadres étatiques et/ou nationaux ne se limite pas aux conséquences de leurs dimensions juridiques à l'échelle mondiale. Leurs modalités de gestion de la diversité interne de leurs sociétés civiles sont aussi exposées par l'effet des spécificités culturelles de leurs sociétés (Bruslé, 2010), des politiques identitaires que les États y mènent (Gillot, à paraître) ou de leurs politiques urbaines (Touré, à paraître).

Le premier type d'effet que nous relevons ici a été illustré, à l'échelle internationale, par la comparaison des complexes commerciaux récréatifs français et mexicains lorsque B. Sabatier, dans sa communication, a signalé la différence de conflictualité entre classes sociales entre les deux États. Mais à l'échelle infra-nationale, en France, cet auteur a pointé également la différence entre les formes spatiales des sociétés locales dijonnaises et paloises, résultant de processus où l'interpénétration de facteurs que l'on peut qualifier de politiques (par exemple, desserte du centre-ville historique par les transports publics à Dijon, à l'inverse de Pau $)^{8}$ avec d'autres, sociodémographiques (composition sociale de la population) ou liés à la morphologie urbaine (Sabatier, 2006), défie la simplicité de l'explication par la seule méthodologie des formations socio-spatiales.

L'espace social est également hiérarchisé. Hiérarchisation et segmentation se combinent dans des situations localisées, concernant parfois des échelles de masse suffisante pour autoriser l'autonomie relative de sociétés locales. S’intéressant aux diverses formes de la hiérarchisation sociale au sein de l'élite coloniale à Saint-Domingue au XVIII siècle, Z. Navarro-Andraud met ainsi à jour « une ligne de fracture à l'aune de la résidence et de la propriété » (Navarro-Andraud, à paraître) entre deux groupes d'administrateurs coloniaux, axée sur le métissage culturel : "Tandis que certains affirment leur identité métropolitaine par un mode de vie résolument urbain et par le refus d'accéder à la propriété dans la colonie, d'autres n’hésitent pas à s'emparer de l'espace rural. Or, cette appropriation du territoire se révèle être également une appropriation du mode de vie local témoignant, de ce fait, de la créolisation des mentalités » (Navarro-Andraud, à paraître). La dialectique de la structuration de l'espace social par l'action des individus qui s'y inscrivent et le remodèlent est illustrée par une remarque de l'auteure : "Il est par ailleurs intéressant de noter que le pouvoir royal avait anticipé ce phénomène de créolisation de ses agents, ainsi qu'en témoignent les ordonnances interdisant aux administrateurs de se porter acquéreur dans les colonies ou de s'y marier sans autorisation royale. Cependant, la monarchie qui règlemente l'accès à la propriété des administrateurs est elle-même à l'origine du contournement de la règle par ces derniers en accordant des passe-droits en fonction des réseaux clientélistes de la cour et des bureaux versaillais »

8. Par l'emploi du terme « facteur » nous assumons ici un «moment pauvre de la recherche » (Lévy, 2003c) qui a néanmoins l'avantage de ne pas considérer le vocabulaire relatif à la qualification des différents sous-ensembles du monde social (Grossetti, 2006) comme définitivement stabilisé et partagé par tous les chercheurs. 
(Navarro-Andraud, à paraître). Les contextes historiques changent. Les motifs des modes de localisation se sont modifiés, tandis que ces modes se sont assouplis et diversifiés, au moins pour une partie de la population mondiale. Mais aujourd'hui encore, les distances physiques ne pouvant être totalement abolies, les parcours sociaux dont notre schéma illustre quelques exemples restent à notre sens scandés par des étapes plus ou moins longues dans des formes spatiales séparées par des discontinuités plus ou moins fortes.

\section{Parcours sociaux}

L’exemple qui précède confirme que la géographie n'est pas la seule discipline intéressée par la description de l'espace social et l'explication de son évolution. Par contre, la manière dont elle étudie aujourd'hui ses entités lui est plus spécifique. Ainsi, les termes d'agent ou d'acteur n'apparaissent pas nécessairement dans toutes les contributions pour qualifier les situations sociales d'individus ou de groupements sociaux. Comparativement, ils sont plus souvent absents et apparaissent moins fréquemment dans les textes des non-géographes. Les géographes, pour leur part, utilisent très souvent le seul terme d'acteur. Le propos vise ici autant à sensibiliser les autres disciplines à cette spécificité de la géographie qu'à engager la clarification de notre posture quant à l'emploi de ces termes.

B. Laplante a explicité dans sa communication une conception des relations entre ces deux termes avec laquelle nous pouvons nous accorder. Analysant des situations d'énonciation des valeurs spatiales de l'habiter émises par des agents immobiliers de la région de Tours ${ }^{9}$, il a réparti ainsi leur qualification sociale : à «l'acteur » la construction de qualification des espaces et d'évaluation des biens, à "l'agent » la participation à la structuration du marché immobilier. Nous refusons cependant la posture qui conduirait à considérer le marché immobilier comme un agencement qui « disparaîtra, en tant que tel, avec la situation précise qui le porte et qu'il soutient, même si un arrangement matériel d'une partie des objets de société mobilisés dans l'action peut perdurer »(Lussault, 2003b). Car les normes (ici, celles du marché immobilier) inscrites dans l'espace social, qui ont permis à l'acteur de développer une action spatiale, évoluent dans une temporalité qui dépasse l'échelle de temps dans laquelle s'inscrit cette action et celle de l'existence de l'acteur, la forme spatiale « région de Tours » constituant ici une des manifestations de la dimension spatiale de ces normes.

Les textes du colloque présentent plusieurs exemples de ce que nous entendons ici par l'expression «parcours social » au sein d'un espace social segmenté (en particulier pour les processus qualifiés de migratoires), dont un cas nous suffira à confirmer la pertinence de cette notion au-delà de notre discipline. Le statut d'étudiant confère ainsi des avantages qui permettent l'exercice d'une « distinction spatiale » (Salane, à paraître) dans le parcours carcéral de cette catégorie d'individus, qu'il s'agisse des déplacements à l'intérieur d'une prison ou d'un établissement pénitentiaire à un autre.

Après cette rapide approche de quelques exemples des types d'êtres et d'états sélectionnés par notre interprétation des textes du colloque, nous poursuivons la lecture de ses apports par une catégorisation de la diversité des formes de collecte des données traitées par leurs auteurs, laquelle propose de distinguer différents niveaux de généralité de l'action spatiale.

\section{UNE GRILLE ANALYTIQUE DE BASE : DISCOURS ET PRATIQUES}

Si la thématique de l'appropriation conduit de manière privilégiée à une analyse des pratiques des formes spatiales, c'est moins le cas de celles de la valorisation et surtout de la catégorisation, qui se manifestent plutôt par des discours sur ces formes. Une lecture des textes du colloque en termes d'épistémologie analytique révèle donc que ceux-ci ont privilégié, soit les discours, soit les pratiques, comme données soumises à interprétation. Cette partition nous sert essentiellement à

9. Laplante B., Interactions et justifications des valeurs spatiales de l'habiter, communication non publiée. 
mettre en évidence l'existence de différents registres et niveaux de construction des valeurs spatiales, des plus généraux aux plus spécifiques, dont notre schéma propose un exemple très simplifié. Quant à la qualification de l'évolution des formes spatiales à laquelle les actions aboutissent, rien ne permet de dire a priori, à cette étape de notre propos, si elle est positive ou négative pour les individus qui les arpentent; c'est pourquoi nous emploierons des termes neutres à cet effet.

\section{Différents types de saisie et de restitution des discours}

Plusieurs textes du colloque utilisent des discours comme données, qu'il s'agisse de discours préalables à l'enquête ou recueillis lors d'observations, d'entretiens... Leurs analyses peuvent révéler le sens attribué par les individus à leurs actions a posteriori, mais aussi les anticipations de ces actions comme dans la communication d'E. Bonerandi basée sur les réponses de Rmistes de Thiérache à un questionnaire portant en particulier sur le seuil de distance envisageable pour leurs déplacements dans le cadre d'une recherche d'emploi ${ }^{10}$.

Les analyses de B. Laplante et d'E. Bonerandi ont montré, même si elles usaient de discours de différents types, que ceux-ci sont des « dispositifs de construction sociale de la réalité qui contribuent à la maintenir, à la renforcer, voire à la subvertir» (Mondada, 2003). Alors que les discours d'agents immobiliers analysés par le premier renforcent les valeurs des biens à la vente desquels ils sont intéressés, le discours scientifique de la seconde propose un renversement des valeurs dans l'explication de la non-mobilité des Rmistes enquêtés. Plutôt que d'y voir la seule résultante d'un déterminisme de disqualification sociale, l'auteure a fait l'hypothèse d'une « stratégie de moins mal être » en comparaison des efforts qu’obligerait la mobilité pour un bénéfice incertain. Cette recherche d'une lecture plus précise de la réalité de l'espace considéré n'ayant pas encore eu d'impact sur les politiques publiques qui contribuent à sa spatialisation, notre schéma laisse ouverte la possibilité d'un relatif isolement des différents types de discours les uns par rapport aux autres.

D'autres contributions confirment, de manière plus directe, le caractère configurant des discours pour l'espace. C'est le cas du discours politique préconisant une disparition du vocabulaire du zonage dans le cas des ZUP (Ratouis, à paraître) et des discours médiatiques émis lors de «la campagne présidentielle française de 2007 dans la presse écrite » (Rivière, à paraître). Deux autres textes analysent des discours de provenances diverses, qu'il s'agisse des « sélections mémorielles différenciées » (Zanetti, ce numéro de Norois) qu’opèrent les différents acteurs participant à la patrimonialisation de la Manufacture d'Armes de Saint-Étienne ou des prescriptions d'aménagement des montagnes méditerranéennes françaises au XIX siècle (Fesquet, à paraître). Ces deux exemples sont regroupés dans notre schéma sous l'appellation « discours d'experts sectoriels », avec celui des agents immobiliers analysé par B. Laplante. Si les positions réciproques des différents types de discours dans ce schéma restent indicatives, réajustables et à compléter selon les contextes d'analyse, insistons sur la dialectique de la stabilisation et de la subversion du spatial par les discours qu'il résume. L'expression discours aménageur y rend compte des décalages possibles entre ce type de discours et les pratiques habitantes qu'a révélés l'analyse par deux urbanistes des projets de réaménagement d’une commune de l'agglomération de Roubaix. Face au discours dominant d'une nécessité de la densification des villes et du renouvellement de la population concernée par le renouvellement urbain pour assurer la mixité sociale, ils ont montré l'intérêt de conserver une diversité typologique des modes d'habiter dans ce lieu pour ne pas y séparer des familles (Couic et Roux, à paraître).

\section{Différents types de saisie et de restitution des pratiques}

Les communications au colloque ont proposé différents types de saisie des pratiques de l'espace, certaines les qualifiant très précisément. Il en est ainsi de la présence ou de l'absence volontaires

10. Bonerandi E., Dévalorisation spatiale, dévalorisation sociale : corrélation ou renversement des valeurs à travers le rapport à la mobilité des Rmistes de Thiérache, communication non publiée. Emmanuelle Bonerandi est malheureusement décédée au début de l'année 2011. 
(hors raisons professionnelles) de certaines classes sociales dans les complexes commerciaux récréatifs (Sabatier, 2006). D'autres textes révèlent plutôt l'organisation des conditions des pratiques d'appropriation de l'espace, cette voie d'analyse pouvant être couplée avec la précédente, comme dans la communication de B. Sabatier qui a évoqué l'effet des formes de sécurisation de ces espaces commerciaux sur leur fréquentation. L'organisation des pratiques par l'aménagement des espaces, à divers niveaux de généralité dont les espaces de travail tels que des ateliers d'usine (Meslin, à paraître) autorise et suscite ainsi des actions de plus ou moins grande portée selon les positions des individus ou des groupes dans l'espace social.

Dans leurs rapports à l'espace, les pratiques sont, malgré leurs différences avec les discours, redevables de la même approche dialectique, d'où la mention dans notre schéma de la stabilisation et de la subversion du spatial par les pratiques. Les tensions politiques liées aux aspects commerciaux des processus d'appropriation du quartier de La Chapelle à Paris par des populations d'origine tamoule témoignent de ces deux types de situations. Dans ce contexte, une partie des « habitants doivent adapter leur comportement aux modifications de leur nouvel environnement (en tout cas, vécu comme nouveau). Ne plus pouvoir faire ses courses de dernière minute à l'alimentation locale, ne plus pouvoir acheter sa baguette au coin de la rue : autant de choses considérées comme normales et propres au mode de vie français qui ne sont désormais plus possibles. Ces faits en apparence anodins sont vécus comme de mini drames » (Dequirez, 2010) au point d'alimenter des discours politiques de lutte contre une dévalorisation supposée du quartier, voire d'exclusion. Mais d'autres habitants s'adaptent volontiers à sa diversification culturelle en cours, anticipant en quelque sorte la stabilisation de la nouvelle forme spatiale, stabilisation déjà consacrée par des discours médiatiques la qualifiant de « quartier tamoul».

À la différence des discours, où les manifestations des contradictions entre stabilisation et subversion du spatial semblent pouvoir rester minoritaires (hypothèse à vérifier), la diversité des types de pratiques évoqués par les textes du colloque nous amène à insister sur leurs ajustements. L'analyse du réaménagement du secteur de Pou de la Figuera à Barcelone met en exergue un de ces exemples d'ajustement, celui entre les objectifs initiaux de rénovation de la municipalité (« désir de replacer le centre ancien dans un contexte métropolitain », H. Ter Minassian, ce numéro) et ceux des associations de résidents mobilisées pour une réhabilitation à l'échelle du quartier (stabilisation du spatial).

Cette approche dialectique de la stabilisation et de la subversion du spatial par les actions, qu'il s'agisse de discours ou de pratiques, permet d'envisager son caractère mobile dans le temps, mais ne précise pas comment traiter des temporalités de l'espace. Sur cette question à peine explorée en géographie nous nous appuierons sur une conception des temporalités prenant ses distances avec tout présentisme ${ }^{11}$ et envisageant la possibilité d'un futur comme anticipation positive (Dubar, 2008). Il s'agit donc d'approfondir les liens entre la notion d'appropriation sociale des formes spatiales et celle de réification de ces formes dans ce qu'elle signifie pour les individus qui les arpentent.

\section{De la stabilité de l'espace et des espaces}

La fécondité de la problématique de l'appropriation de l'espace a été confirmée par la diversité des contributions au colloque de Caen. Ceci étant, il est possible que l'intérêt heuristique de cette notion se heurte à une limite, celle précisément de l’absence de limite envisageable de son application à tout phénomène ; après le tout-territoire, l'usage incontrôlé de la notion de territoire dans la géographie française (Séchet et Keerle, 2009), y existe-t-il un risque du tout-appropriation? La réponse à cette question passe en particulier par la ré-interrogation des intentions des promoteurs de la notion. Pour F. Ripoll et V. Veschambre, « raisonner en terme d'appropriation

11. Le présentisme est un mode collectif de rapport au temps, un « régime d'historicité » (Hartog, 2003) qui privilégie le présent par rapport au passé, au futur, ou à une eschatologie qui en ferait une parenthèse. 
présente $[\ldots]$ plusieurs intérêts méthodologiques et théoriques majeurs [...] [dont celui] de mettre l'accent sur le social et sa dimension spatiale plutôt que sur l'espace lui-même, [...] d'appréhender les acteurs et leurs pratiques, les rapports et les processus sociaux plutôt que des "objets géographiques" trop souvent réifiés voire personnifiés » (Ripoll et Veschambre, 2005). Même si la critique de la personnalisation des espaces reste nécessaire en géographie, il semble que le débat s'y soit récemment déplacé, de manière complémentaire, quant à l'usage du terme de réification, à propos de la qualification des processus sociaux dont notre discipline explore la dimension spatiale $^{12}$. Nous arguerons donc de la possibilité d'échapper à une conception réifiée des formes spatiales par l'introduction d'une réflexion sur les temporalités des processus sociaux qui les produisent. Cette réflexion nous conduira finalement à une analyse des liens entre changements des formes spatiales et changements des rapports sociaux collectifs ou individualisés à ces formes. La prise en compte du temps dans l'action, engagée précédemment par l'intermédiaire de la notion de parcours social, sera reliée à ses spatialités. Notre posture théorique en géographie sociale sera ainsi complètement explicitée.

\section{RÉIFICATION DU SPATIAL}

La longue suprématie de l'analyse des formes sur celle des processus en géographie semble avoir déclenché un mouvement récent en retour, certes régénérant, mais à notre sens excessif, de dévalorisation de l'interrogation sur les formes spatiales. La communication d'un auteur suisse (M. Schaffter), portant sur la catégorisation des objets géographiques et basée sur l'exemple du couple ville-campagne, est apparue plus nuancée sur cette question. En considérant que « les enjeux qui justifient l'acte de catégoriser, le lieu où celui-ci se produit, ainsi que la personne qui l'effectue sont autant d'éléments qui font varier les modalités de catégorisation » (Giacomel et Schaffter, à paraître), il laisse ouverte la question de la prise en compte de la forme spatiale comme contexte matériel de l'action. Notre interprétation de cette posture ne limite pas la pratique de la géographie à un « réalisme dialogique [qui] reconnaît la liaison permanente de l'idéel et du matériel » (Lussault, 2003c) mais affirme, pour sa partie matérielle, un réalisme transcendental (Vandenberghe, 1998). Dans cette conception, le monde matériel « existe indépendamment des observations et des descriptions que nous pouvons en faire - ce qui n'implique pas, bien sûr, que nous puissions le connaître indépendamment de ces observations ou descriptions » (Vandenberghe, 1998). Il existe alors des critères (importance de l'agriculture, densité de l'habitat...) qui permettent d'objectiver la catégorisation des espaces en termes de campagne ou de ville, par exemple. Par contre, les idées de campagne, de ville ou relatives à toute autre forme de spatialisation des processus sociaux dépendent des « concepts qui flottent dans la tête des hommes et orientent leurs actions " (Vandenberghe, 1998). La question est alors de savoir à quelles théories il est possible de recourir en sciences anthroposociales pour expliquer la production des formes spatiales et des valeurs qui leurs sont attribuées, ainsi que la manière dont ces formes orientent l'action.

À notre sens, les formes spatiales, comme les structures sociales, préexistent à l'action des individus. Cette conception de la dimension spatiale du social diffère de celle exposée dans l'article «valeur spatiale » du Dictionnaire de la géographie et de l'espace des sociétés qui évoque à propos de la valeur immobilière un « ensemble : prix + qualification discursive conventionnelle, réifié par les protagonistes eux-mêmes et qui apparaît de ce fait aux yeux de tous comme extérieur aux personnes » (Lussault, 2003a). Car ce qui est ici signalé comme une réification constitue plutôt une chosification méthodologique ou, plus simplement, une objectivation (Vandenberghe, 1997): les formes de l'habitat présentent à l'observation des valeurs immobilières, certes activées et rema-

12. Les emplois successifs du terme « réification » dans les articles relatifs à Karl Haushofer et à la géographie historique dans le Dictionnaire de la géographie et de l'espace des sociétés illustrent cette évolution du débat (Staszak, 2003a et b). 
niées par des acteurs individuellement identifiables à un moment considéré, mais qui résultent de processus sociaux $^{13}$ dont la spatialisation s'est matérialisée dans l'étendue avant leur intervention.

Il est donc nécessaire de maintenir au sein du dispositif de connaissance de la géographie une orientation de recherche relative à l'analyse des formes spatiales afin de mieux préciser leur statut dans la reproduction et la dynamique sociales. Dans leur étude de l'évolution récente du $19^{\text {e }}$ arrondissement de Paris, M. Guérois et C. Hancock ont ainsi montré que des espaces de la capitale, très proches dans leur morphologie urbaine et la composition sociale de leurs habitants de ceux des communes de banlieue limitrophes, faisaient l'objet d'une valorisation immobilière supérieure à celle de ces derniers, laquelle s'explique à la fois par les représentations sociales plus favorables de l'espace parisien, les capacités d'investissement de la municipalité parisienne en équipements sociaux et l'impact du quadrillage policier (d'origine nationale) limitant les dégradations de ces espaces (Guérois et Hancock, à paraître).

Pour que l'identification scientifique de cette chosification des formes spatiales par les acteurs sociaux, qui constitue une première étape de l'analyse, ne se transforme pas en réification sociale pour la géographie, c'est-à-dire en absence de mise en perspective critique de l'émergence et du devenir de ces espaces, plusieurs voies sont possibles. Une de ces voies est l'interrogation des politiques publiques sous l'angle de la justice (Séchet et al., 2011). C'est ce type de problématique qu'ont illustré M. Guérois et C. Hancock (à paraître) en questionnant la politique de la municipalité parisienne, laquelle mise sur une gentrification partielle de l'espace concerné tout en ne remettant pas en cause l'existence de son parc de logement social. Cette voie permet à la géographie d'échapper à la réification sociale pour sa qualification de contextes d'actions relativement limités dans le temps. L'exploration d'une autre voie, complémentaire, est nécessaire pour que la géographie puisse éviter son instrumentalisation par la naturalisation des valeurs spatiales lorsqu'elle traite de formes spatiales plus stables dans le temps.

\section{TEMPoralités ET SPATIALitÉS}

Cette démarche, qui concerne les temporalités des formes spatiales, suppose que la géographie, dans ses pratiques d'objectivation de ces formes, choisisse de systématiser l'expression du fait que « les activités sociales produisent, chacune ou presque, des temporalités spécifiques » (Di Méo et Buléon, 2005). Il ne s'agit pas pour autant de contraindre systématiquement le langage d'exposition des données à une prise en compte des temporalités qui transformerait les géographes en historiens mais à les inciter à situer dans le temps l'émergence des valeurs données aux formes spatiales dans l'espace social, et le cas échéant, leurs phases de recomposition. Le texte de T. Pfirsch illustre bien l'enjeu de cette orientation intradisciplinaire, condition de la possibilité d'une prise en compte non réifée des formes spatiales. Il montre que les vieilles familles d'origine rurale de la bourgeoisie napolitaine, qui étaient parvenues à conserver leur maison de famille rurale, élément d'un système multirésidentiel hérité, « grâce à des pratiques successorales restées très inégalitaires jusque dans les années 1950 » (Pfirsch, 2010), sont aujourd'hui confrontées à la difficulté de la transmission de ces maisons. En déclin économique, amenées à s'adapter à la mutation spatiale

13. Ces processus ayant été construits précédemment par d’autres acteurs. La philosophie réaliste (anglophone) s'adresse à la fois aux sciences naturelles (réalisme transcendantal) et aux sciences sociales (réalisme critique). En sociologie, elle aboutit à considérer que les structures sociales sont aussi réelles que les individus qui les reproduisent ou les transforment, mais que ces deux éléments «ne sont pas co-variants avec le temps» (Archer, 2004, p. 333), ce qui permet de reconsidérer les sens respectifs des termes « acteur» et «agent». Dans cette perspective, l'individu est d'abord un agent, du fait de la position sociale que les structures sociales lui permettent d'occuper avant de, peut-être, exercer de manière personnelle et volontaire le rôle d'un acteur social dont le succès de l'action dépendra des effets des actions collectives des groupes d'agents définis par les structures sociales et au sein desquels il est positionné. C'est la réflexivité de la personne qui lui permet de devenir un agent actif et un acteur interagissant, et donc d'échapper à la réification, si toutefois cette réflexivité est suffisamment développée (Archer, 2003). Rappelons que « globalement, la réification sociale a trait au fonctionnement relativement autonome, aliéné et aliénant, des systèmes de la culture et de la société modernes et à leur transformation de moyens en fins pour soi » (Vandenberghe, 1997). 
des lieux de villégiature et de sociabilité des autres élites urbaines et ceci dans un cadre de diffusion des successions égalitaires, elles doivent choisir entre « conserver leur maison de famille en en changeant les usages » (Pfirsch, 2010), ou « disséminer ses fonctions dans l'espace patrimonial en la transférant partiellement vers d'autres lieux hérités » (ibid.).

Les contributions au colloque présentent, selon leurs problématiques respectives, une prise en compte variable de la dimension temporelle des spatialisations. Celles des géographes se distinguent souvent, à l'inverse de ceux des auteurs d'autres disciplines, par l'accent mis sur des changements de l'espace ou des espaces plus que sur des changements de spatialités humaines. Cependant, ces deux notions sont mêlées : si nous considérons avec M. Lussault que la spatialité humaine est « une réalité construite dans l'action spatiale et qui signifie quelque(s) chose(s) pour quelqu'un » (Lussault, 2003d), ce quelqu'un n'est ni isolé socialement ou spatialement, ni inscrit dans une temporalité limitée au présent (Gislain, 2004). Et là encore, s’il n'y a ni acteur omnipotent, ni agent totalement inhabile dans l'action, les résultats des actions, analysés dans la perspective du réalisme critique, révèlent que dans un laps de temps donné, les individus voient leurs capacités d'action s'accroître ou diminuer. Certes, le volume des ressources mises en jeu dans ces actions n'est pas «à somme nulle »(Lévy, 2003b), mais la possibilité d'accroître « la quantité d'espace présente dans une société » (Lussault, 2003d) n'est pas non plus infinie. L'espace matériel est donc un enjeu et l'inégale capacité d'usage par les individus de cet espace reste dépendante de leurs positions sociales.

Le texte de D. Goeury illustre ce lien entre changements de l'espace, changements d'espaces et changements de spatialités humaines. Il montre que la spécificité physique des hautes vallées du Haut-Atlas central au Maroc et de l'ouest de l'Himalaya indien les a d'abord transformées en enclaves de l'espace de la société industrielle mondialisée, donc en " espaces du mérite » (Goeury, à paraître) inventés comme tels par des touristes-voyageurs-aventuriers. Les spatialités des premières petites cohortes de touristes qui leur succèdent se traduisant par une aide au développement local de ces espaces, leur désenclavement progressif conduit maintenant à la fois à l'augmentation de leur fréquentation touristique, stimulée par des politiques nationales, et au remplacement des touristes les plus aventuriers de la première génération par un tourisme en voie de massification.

La contribution d'un des anthropologues représentés au colloque permet de différencier la géographie de la spatiologie, expression employée par C. Chivallon (2008) pour qualifier une orientation de recherche transdisciplinaire (dans un premier temps) qui exclut logiquement de son propos la question de l'analyse des spécificités matérielles des formes spatiales (parmi lesquelles leur situation par rapport à d'autres formes). Dans son analyse de l'impact sur l'appropriation de l'espace de la culture du cannabis chez les Ketama du Rif central au Maroc ${ }^{14}$, cet auteur s'intéresse très précisément aux changements de spatialités de ses habitants qui en ont tiré profit, mais pas aux changements de position de cet espace dans l'espace mondial ou national. Ce choix d'analyse, s'il est pertinent dans le langage disciplinaire de son auteur, ne peut être adopté en géographie sans risque de réduction de cette spatialité au lieu de résidence vécu au présent. C'est ce que montre la discussion par une géographe des modalités de la description de la spatialisation des enfants de la rue à Yaoundé (Morelle, à paraître). Limiter la description d'une forme spatiale aux formes de son appropriation par une seule partie des individus qui s'y spatialisent sans prendre en compte leurs parcours sociaux et sans tenir compte de la cospatialité (Lévy, 2003d) de cette forme avec d'autres conduit à la considérer comme une entité isolée ne faisant l'objet d'aucune contradiction dans ses usages. Alors que si l'on considère la spatialité comme la dimension spatiale d'actions sédimentées en structures et d'actions qui les transforment, il paraît possible d'éviter des disjonctions d'analyse de réalités sociales fonctionnant dans des temporalités différentes. Ainsi, le débat sur le séparatisme spatial qui a incité la proposition de la spatiologie nous semble

14. Mouna K., La culture du kif et son impact sur l'appropriation de l'espace : le cas de Ketama dans le Rif central (Maroc), communication non publiée. 
devoir être poursuivi dans de nouveaux termes, la différence entre «spatial» (structures sociales sédimentées) et «social » (action contemporaine sur les structures) nous paraissant redevable de la prise en compte différenciée des temporalités sociales dans l'analyse.

\section{Conclusion}

Ce propos n'a pas vocation à se substituer à chacun des textes de présentation des différents supports de publication des contributions au colloque de Caen. Il souhaite inviter à la lecture de ces contributions, dont notre choix d'interprétation ne pouvait rendre compte de tous les motifs à intérêt, et inviter leurs auteur-e-s à poursuivre les dialogues entre disciplines entrevus lors de ce colloque.

Les espaces analysés dans ces contributions présentent une grande diversité d'échelles spatiales et temporelles. En utilisant pour les qualifier l'expression formes spatiales, plutôt que celle d'agencements spatiaux, nous avons souhaité éviter de marquer une rupture radicale avec un héritage disciplinaire dont il paraît plus fécond de continuer à analyser les errements que de risquer de les reproduire par son oubli. Dans le même temps, nous souhaitions aussi éviter d'accorder de manière générale un rôle globalement plus important aux actions d'innovations qu'aux actions de reproduction sociale dans la production de l'espace, les situations concrètes pouvant d'ailleurs être très diverses à cet égard : un centre commercial récréatif offre plus de prises à l'innovation pour ceux qui le fréquentent régulièrement qu'un atelier d'usine. Notre positionnement quant à la notion de réification paraît le moyen le plus sûr de concilier ces deux approches de la dynamique du social.

Cette posture critique n'exclut pas la possibilité d'une analyse objective de la dimension spatiale des sociétés. En invitant à concevoir les processus de spatialisation de la valeur sous l'angle de certains de leurs enjeux sociaux, le colloque a favorisé l'expression d'analyses mettant en valeur la géographie sociale, ou, issues d'autres disciplines, susceptibles d'entrer en dialogue avec elle. C'est dans la perspective de ce dialogue que nous avons réduit la description des modalités de la spatialisation des rapports sociaux à des catégories très générales. Notre proposition d'une appréhension de l'espace comme dimension de la société révélant les dynamiques de sa reproduction par le biais d'actions conduisant à stabiliser ou à subvertir les formes de leurs contextes rend possible la mise en évidence des contradictions entre ces actions ainsi qu'entre les résultats attendus par les acteurs et révélés par leurs parcours. Elle évite la réduction des processus de reproduction de la société aux phénomènes de domination qui ne constituent qu'une de leurs caractéristiques tout en ne préjugeant pas de l'avenir des formes de coordination de l'action entre individus et des modalités d'organisation de l'espace dont les sciences anthroposociales explorent les possibilités.

La confrontation de notre interprétation des apports du colloque avec des efforts de formalisation de la discipline élaborés dans le Dictionnaire de la géographie et de l'espace des sociétés nous a permis de préciser nos convergences et nos divergences avec certaines des définitions qui y sont proposées. Notre recours à l'appellation géographie sociale, effectué dans le prolongement de travaux qui s'en réclament, a mis l'accent sur sa distinction face à des définitions de la discipline excluant de leurs objectifs les nécessaires articulations interdisciplinaires de la connaissance. Il persistera tant que ne sera pas admise en géographie la posture du pluralisme explicatif face à toute norme émanant d'une théorie intégratrice ou d'un bornage disciplinaire.

Pour autant, la pratique de la géographie dans une perspective interdisciplinaire, qu'elle prenne ou non la forme d'un nouveau cadre d'analyse (comme la « spatiologie ») ne nous paraît pas devoir rendre caduque les analyses des formes spatiales focalisées sur leur matérialité et incluant l'espace terrestre comme une de leurs échelles de référence. Là comme ailleurs, c'est surtout la réification qui doit être évitée, ce que le colloque de Caen nous aura permis de préciser. 


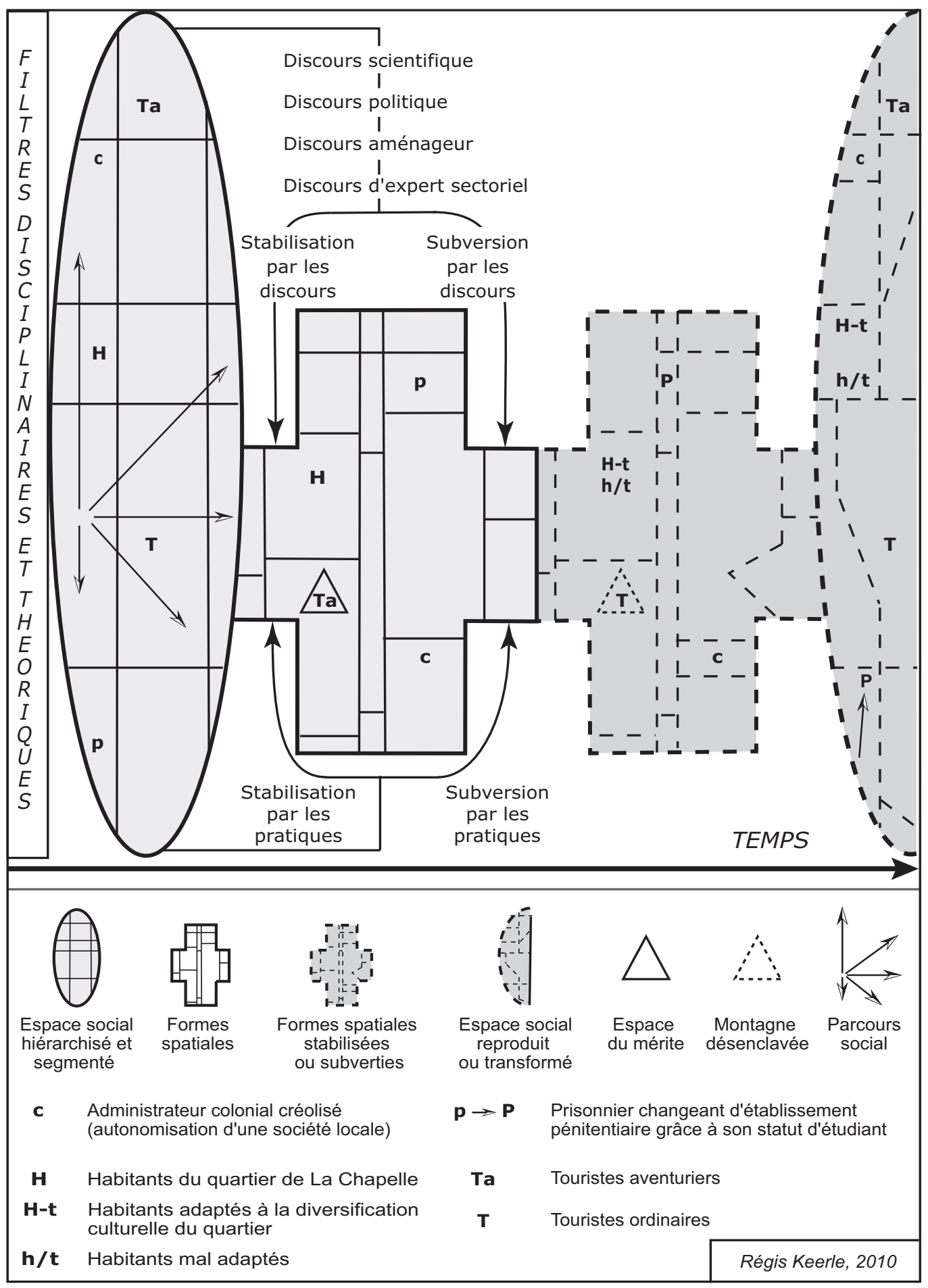

Figure 1 : Reproduction et transformation de l'espace social et des formes spatiales par les actions Reproduction and transformation of social structure and spatial objects by agency 


\section{Liste des textes présentés au colloque de Caen et en voie de publication}

Couic M.-C., Roux J.-M., Les Hauts Champs de Hem, un «Chelsea du pauvre »? La maison et la barre. Antagonismes et complémentarités des formes d'habiter dans un quartier d'habitat social, dans Backouche I., Ripoll F., Tissot S., Veschambre V. (dir.), à paraître. Espaces hérités, espaces enjeux. Sciences sociales en débats (titre provisoire), Rennes, PUR.

Debroux T., Decroly J.-M., Deligne C., Galand M., Loir C., Van Criekingen M., Permanence et transformation des espaces résidentiels élitaires à Bruxelles (xvIII ${ }^{\mathrm{e}}$-XXI ${ }^{\mathrm{e}}$ siècle), dans BACKouche I., RipolL F., Tissot S., Veschambre V. (dir.), à paraître. Espaces hérités, espaces enjeux. Sciences sociales en débats (titre provisoire), Rennes, PUR.

Dumasy F., Au milieu et à part. Prestige et centralité à tripoli de Libye pendant la colonisation italienne, 1911-1943, dans Backouche I., Ripoll F., Tissot S., Veschambre V. (dir.), à paraître. Espaces hérités, espaces enjeux. Sciences sociales en débats (titre provisoire), Rennes, PUR.

FEsquet F., Espaces produits, espaces prescrits : formes et enjeux du contrôle des espaces montagnards. L'exemple des montagnes méditerranéennes françaises au XiX ${ }^{\mathrm{e}}$ siècle, dans Backouche I., Ripoll F., Tissot S., Veschambre V. (dir.), à paraître. Espaces hérités, espaces enjeux. Sciences sociales en débats (titre provisoire), Rennes, PUR.

FLeury C., Frontière marine et espace social : l'appropriation de la mer dans le golfe Normand-Breton.

Gillot L., Conflits et inégalités sociales associés à l'appropriation des sites historiques habités : le cas de Bosra en Syrie, dans Backouche I., Ripoll F., Tissot S., Veschambre V. (dir.), à paraître. Espaces hérités, espaces enjeux. Sciences sociales en débats (titre provisoire), Rennes, PUR.

Goeury D., Les espaces du mérite : enclavement et construction d'un espace transnational, dans BACKOuCHE I., Ripoll F., Tissot S., Veschambre V. (dir.), à paraître. Espaces hérités, espaces enjeux. Sciences sociales en débats (titre provisoire), Rennes, PUR.

Guérois M., Налсоск C., Vendre le $19^{\mathrm{e}}$ arrondissement de Paris : dé-qualification et re-classement d'espaces périphériques de la capitale, dans Backouche I., Ripoll F., Tissot S., Veschambre V. (dir.), à paraître. Espaces hérités, espaces enjeux. Sciences sociales en débats (titre provisoire), Rennes, PUR.

Guılzot F., Conflit pour la terre et lutte de légitimité. Réflexions sur l'appropriation à partir de l'exemple du mur de séparation entre israéliens et palestiniens, dans Backouche I., Ripoll F., Tissot S., Veschambre V. (dir.), à paraître. Espaces hérités, espaces enjeux. Sciences sociales en débats (titre provisoire), Rennes, PUR.

Mestin K., Espace fragmenté, ouvriers divisés. Les ouvriers de la haute technologie en prise aux segmentations de l'espace de production, dans Backouche I., Ripoll F., Tissot S., Veschambre V. (dir.), à paraître. Espaces hérités, espaces enjeux. Sciences sociales en débats (titre provisoire), Rennes, PUR.

Morelle M., La production de la rue. Appréhension d'une catégorie sociale dans ses dimensions spatiales : les enfants des rues à Yaoundé (Cameroun), dans Backouche I., Ripoll F., Tissot S., Veschambre V. (dir.), à paraître. Espaces hérités, espaces enjeux. Sciences sociales en débats (titre provisoire), Rennes, PUR.

Ratouis O., Énoncer, découper, véhiculer. ZUP ou les variations d’une catégorie déshéritée, dans Backouche I., Ripoll F., Tissot S., Veschambre V. (dir.), à paraître. Espaces hérités, espaces enjeux. Sciences sociales en débats (titre provisoire), Rennes, PUR.

Rivière J., Catégorisations des espaces et (dé)valorisations des habitants dans les discours médiatiques. La campagne présidentielle française de 2007 dans la presse écrite, dans Backouche I., Ripoll F., Tissot S., Veschambre V. (dir.), à paraître. Espaces hérités, espaces enjeux. Sciences sociales en débats (titre provisoire), Rennes, PUR.

Salane F., Être étudiant en prison : la «distinction spatiale », dans Backouche I., Ripoll F., Tissot S., Veschambre V. (dir.), à paraître. Espaces hérités, espaces enjeux. Sciences sociales en débats (titre provisoire), Rennes, PUR.

TourÉ M., Acteurs et pratiques de la gestion communale bamakoise : tensions et clivages entre normes et stratégies citadines, dans Backouche I., Ripoll F., Tissot S., Veschambre V. (dir.), à paraître. Espaces hérités, espaces enjeux. Sciences sociales en débats (titre provisoire), Rennes, PUR. 
VermeErsch S., Les couches moyennes contemporaines : mosaïque sociale, mosaïque spatiale, dans BACkouche I., Ripoll F., Tissot S., Veschambre V. (dir.), à paraître. Espaces hérités, espaces enjeux. Sciences sociales en débats (titre provisoire), Rennes, PUR.

Pfinsch T., 2010. Maisons de famille et systèmes de villégiature dans les élites urbaines : l'exemple de Naples, Genèses, n 81, p. 104-127.

Mazaud C., 2010. Le rôle du capital d'autochtonie dans la transmission d'entreprises artisanales en zone rurale, Regards sociologiques, $n^{\circ} 40$, p. 45-57.

Bruslé B., 2010. Rendre l'étranger familier. Modes d'appropriation et de catégorisation de l'espace par les migrants népalais en Inde, Revue Européenne des Migrations Internationales, vol. 26, n 2, p. 77-94.

Dequirez G., 2010. Processus d'appropriation et luttes de représentation dans le « Little Jaffna » parisien, Revue Européenne des Migrations Internationales, vol. 26, n² 2, p. 95-116.

- Sources complémentaires aux communications dont la publication est différée

Giacomel G., Schaffter M., à paraître. Quelle pertinence pour les catégories «ville» et « campagne» dans les discours contemporains autour de projets d'aménagement? L'exemple du Piano di Magadino (canton du Tessin, Suisse), Actes du colloque "Les périphéries urbaines entre normes et innovations : les villes du sud de l'Europe », Bordeaux 11-14 juin 2008; communication : SCHAFFter M., La catégorisation des objets géographiques dans les discours des acteurs de l'aménagement du territoire : autour du couple ville-campagne.

Sabatier B., 2006. La publicisation des espaces de consommation privés. Les complexes commerciaux récréatifs en France et au Mexique, thèse de doctorat; communication : les complexes commerciaux récréatifs, analyseurs de l'appropriation et de la représentation des espaces urbains privés et publics en France et au Mexique, université de Toulouse-Le Mirail, 476 p.

\section{Bibliographie}

Archer M., 2003. Structure, agency and the internal conversation, Cambridge, Cambridge University Press, $370 \mathrm{p}$.

Archer M., 2004. Entre la structure et l'action, le temps. Défense du dualisme analytique et de la perspective morphogénétique [Traduction de Archer M., 1995. Realist Social Theory: The Morphogenetic Approach, Cambridge, Cambridge University Press, p. 65-92], Revue du MAUSS, n² 24, p. 329-350.

Berthelot J.-M., 1996. Les vertus de l'incertitude. Le travail de l'analyse dans les sciences sociales, Paris, PUF, $271 \mathrm{p}$.

Berthelot J.-M., 2001. Programmes, paradigmes, disciplines : pluralité et unité des sciences sociales, dans Berthelot J.-M. (dir.), Épistémologie des sciences sociales, Paris, PUF, p. 457-519.

Bozon M., 1984. Vie quotidienne et rapports sociaux dans une petite ville de province, Lyon, PUL, 300 p.

Bozon M., Сhamboredon J.-C., 1980. L'organisation sociale de la chasse en France et la signification de la pratique, Ethnologie française, $\mathrm{n}^{\circ} 1$, p. 65-88.

Chivallon C., 2008. L’espace, le réel et l'imaginaire : a-t-on encore besoin de la géographie culturelle?, Annales de géographie, $\mathrm{n}^{\circ}$ 660-661, p. 67-89.

Di Méo G., 1987. Objectivation et représentations des formations socio-spatiales, Annales de géographie, $n^{\circ} 537$, p. 564-594.

Di méo G., Buléon P., (dir.), 2005. L'espace social : une lecture géographique des sociétés, Paris, Armand Colin, 303 p.

Dubar C., 2008. Temporalité, temporalités : philosophie et sciences sociales, Temporalités [En ligne], $n^{\circ} 8$, mis en ligne le 9 juillet 2009. [http://temporalites.revues.org/index137.html]. 
Gislain J.-J., 2004. Futurité et toposité : sitologie des perspectives de l'action, Géographie, Économie, Société, $n^{\circ}$ 6, p. 203-219.

Grossetтi M., 2006. Trois échelles d'action et d'analyse. L'abstraction comme opérateur d'échelle, L'Année Sociologique, $\mathrm{n}^{\circ} 2$, p. 285-307.

Hantog F., 2003. Régimes d'historicité : présentisme et expériences du temps, Paris, Seuil, 257 p.

LÉvy J., 2003a. Substance, dans LÉvy J., Lussault M. (dir.), Dictionnaire de la géographie et de l'espace des sociétés, Paris, Belin, p. 880-881.

Lévy J., 2003b. Domination, dans LÉvy J., Lussault M. (dir.), Dictionnaire de la géographie et de l'espace des sociétés, Paris, Belin, p. 277-278.

LÉVy J., 2003c. Factorielle (Approche), dans LÉvy J., Lussault M. (dir.), Dictionnaire de la géographie et de l'espace des sociétés, Paris, Belin, p. 359.

LÉvy J., 2003d. Cospatialité, dans LÉVy J., Lussault M. (dir.), Dictionnaire de la géographie et de l'espace des sociétés, Paris, Belin, p. 213-214.

LÉvY J., 2008. La géographie culturelle a-t-elle un sens?, Annales de géographie, nº 660-661, p. 27-46.

Lussault M., 2003a. Valeur spatiale, dans LÉvy J., Lussault M. (dir.), Dictionnaire de la géographie et de l'espace des sociétés, Paris, Belin, p. 973-974.

Lussault M., 2003b. Agencement, dans Lévy J., Lussault M. (dir.), Dictionnaire de la géographie et de l'espace des sociétés, Paris, Belin, p. 45-47.

Lussault M., 2003c. Matériel/Idéel, dans Lévy J., Lussault M. (dir.), Dictionnaire de la géographie et de l'espace des sociétés, Paris, Belin, p. 592-593.

Lussault M., 2003d. Spatialité, dans Lévy J., Lussault M. (dir.), Dictionnaire de la géographie et de l'espace des sociétés, Paris, Belin, p. 866-868.

Mondada L., 2003. Discours, dans LÉVy J., Lussault M. (dir.), Dictionnaire de la géographie et de l'espace des sociétés, Paris, Belin, p. 264-265.

Ripoll F., Veschambre V., 2005. L'appropriation de l'espace comme problématique, Norois, n 195, p. 7-15.

Séchet R., Keerle R., 2009. Petite histoire des délicatesses de «l'équipe-de-géographie-sociale-de-laFrance-de-l'Ouest » avec le territoire, dans VAnier M. (dir.), Territoires, territorialité, territorialisation. Controverses et perspectives, Rennes, PUR, p. 83-93.

Séchet R., David O., Keerle R., 2011. Penser la justice pour penser les politiques territorialisées. Une application à partir des cas du sport et de la petite enfance, dans Dufaux F., Philifert P. (dir.), Justice spatiale et politiques territoriales, Presses Universitaires de Paris-Ouest, Nanterre.

Staszak J.-F., 2003a. Haushofer Karl, dans Lévy J., Lussault M. (dir.), Dictionnaire de la géographie et de l'espace des sociétés, Paris, Belin, p. 447.

Staszak J.-F., 2003b. Historique (Géographie), dans LÉvy J., Lussault M. (dir.), Dictionnaire de la géographie et de l'espace des sociétés, Paris, Belin, p. 465-466.

Ter Minassian H., 2010. La réhabilitation du centre ancien de Barcelone : multiplicité des enjeux, des réappropriations, des tensions, Norois, p. 13-27.

Vandenberghe F., 1997. Une histoire critique de la sociologie allemande Aliénation et réification (Tome I): Marx, Simmel, Weber, Lukacs, Paris, La Découverte et Syros, 296 p.

VANDENBERghe F., 1998. Une histoire critique de la sociologie allemande Aliénation et réification (Tome II): Horkheimer, Adorno, Marcuse, Habermas, Paris, La Découverte et Syros, 383 p.

Zanetti T., 2010. La Manufacture d’Armes de Saint-Étienne. Un conflit mémoriel, Norois, p. 41-55.

Cet article a été reçu le 15 avril 2010 et définitivement accepté le 13 décembre 2010. 